\title{
Dó, Ré, Mergesort: um relato de experiência interdisciplinar de ensino de computação com matemática e música
}

\section{William Fabian Machado Vera, André Melhor Miranda, Wallyson Nascimento Costa, Ecivaldo de Souza Matos}

\author{
Departamento de Ciência da Computação - Universidade Federal da Bahia (UFBA) \\ Av. Adhemar de Barros, s/n - CEP 40170-110 - Salvador - BA - Brasil \\ \{williamfabianmv, andremelhor, wallycostta\}@gmail.com, ecivaldo@ufba.br
}

\begin{abstract}
This paper presents an experience report concerning of programming teaching with musical and mathematical elements to teach a sorting algorithm (Mergesort) to high school students. It attempted to identify as the musical reasoning could contribute to computational thinking development by subjects in their first contacts with computer programming. The main goal was to identify potential benefits of interdisciplinarity to teach computer science.
\end{abstract}

Resumo. Este artigo apresenta um relato de experiência piloto de ensino de programação. Utilizou-se nesta experiência uma abordagem com uso de elementos do raciocínio musical para o ensino de ordenação (algoritmo Mergesort) para estudantes do ensino médio. Buscou-se identificar, em uma experiência pontual, como o raciocínio musical poderia contribuir para a construção do raciocínio computacional por sujeitos em seus primeiros contatos com a programação de computadores. Serão apresentados os resultados iniciais, com uma turma de curso livre introdutório à programação, ofertada a estudantes do ensino médio, ainda não conclusivos, com o objetivo de identificar potenciais benefícios à prática de ensino de computação interdisciplinar.

\section{Introdução}

Posturas metodológicas interdisciplinares têm sido incentivadas nos currículos escolares. Não é diferente para o ensino de Computação na escola, ou ainda, fora dela. Uma das possíveis formas de assumir postura metodológica interdisciplinar é com o relacionamento de conteúdos com alusão ao contexto dos estudantes, com elementos facilmente reconhecidos por eles.

Posturas metodológicas interdisciplinares agregam valor inestimável aos conhecimentos que se deseja construir (Fazenda, 2010; Warschauer, 2000; Sancho, 2006). Esses estudos, em diferentes momentos, já indicavam a necessidade de ações pedagógicas mais próximas da realidade, conjugadas a uma estratégia de formação humana de caráter teórico-prático, para construção significativa de conhecimentos.

Freire (1996) lembra que a abordagem didática deve considerar a realidade dos estudantes, incentivando-os a se apropriarem do conhecimento e desenvolver a curiosidade epistemológica. É essa forma especial de curiosidade que direciona o estudante a tentar conhecer e reconhecer os elementos do mundo, sintoniza-se com um dos elementos mais 
V Congresso Brasileiro de Informática na Educação (CBIE 2016)

Anais dos Workshops do V Congresso Brasileiro de Informática na Educação (CBIE 2016)

atuais de discussão sobre o ensino de computação: o raciocínio (ou pensamento) computacional.

Nesse sentido, este artigo apresenta um relato de experiência piloto de ensino de programação com uso de elementos do raciocínio musical para o ensino de ordenação (algoritmo Mergesort) para estudantes do ensino médio. Considerando a hipótese de que a associação de conceitos de música aos conceitos computacionais pode favorecer o aprendizado de Computação por escolares, essa ação teve por objetivo identificar, em uma experiência pontual, como o raciocínio musical poderia contribuir para a construção do raciocínio computacional por sujeitos em seus primeiros contatos com a programação de computadores.

A seguir será apresentada a interdisciplinaridade como prática didática de Computação, ressaltando a necessidade da reflexão sobre a construção do raciocínio computacional em escolares e indicando o caráter interdisciplinar de ensino como fator estimulante para tal.

\section{Interdisciplinaridade como prática didática de Computação}

Ribeiro et al (2014) sinalizam para a necessidade de abordar elementos de raciocínio computacional (computational thinking) na educação escolar, dada a complexidade dos elementos de computação, os quais precisam ser manipulados e apropriados pelos sujeitos, exigindo do indivíduo habilidades que vão para além do mero uso das tecnologias, mas na produção e no seu uso consciente. Outros trabalhos têm discutido e apresentado elementos para reflexão sobre a importância da construção do raciocínio computacional na educação básica, como um mecanismo pedagógico articulador, seja com o mundo, seja com outras disciplinas escolares (França e Amaral, 2013; Paiva et al, 2015).

Alguns estudos e relatos de experiência têm refletido sobre a efetividade das práticas atuais de ensino de computação na escola e sobre como reduzir a fragmentação no processo de construção de conhecimento para o estudante que chega à escola com múltiplas experiências no uso de Tecnologias Digitais de Informação e de Comunicação (TDIC), mas pouca articulação entre as competências básicas de análise, síntese e abstração (Paiva et al., 2015).

A abstração é um elemento fundamental para a Ciência da Computação, que tradicionalmente tem-se articulado com áreas como Matemática, Estatística, Física e Engenharias. Considerando a importância dos estudos interdisciplinares na Ciência da Computação, para sua evolução enquanto ciência e aplicação prática para solucionar os mais complexos problemas sociais (Cassel, 2011), novas iniciativas de integração epistemológica têm sido estudadas (Souza, 2016; Matos, Paiva e Corlett, 2016).

Considerando a Computação como mais uma linguagem, muitas vezes tratada com as mesmas complexidades de aprendizado das línguas estrangeiras (Hirotaka, 2014), talvez seja possível analisar a dinâmica do processo dialético de pseudoconfronto entre as linguagens, numa perspectiva de estabelecimento de possibilidades intersemióticas (Plaza, 1987); além de problematizar conflitos e/ou soluções criativas nas interlocuções entre as mais diversas linguagens que permeiam o nosso cotidiano, influenciando diretamente as políticas e ações de formação humana, em nível macro (propostas curriculares, parâmetros 
V Congresso Brasileiro de Informática na Educação (CBIE 2016)

Anais dos Workshops do V Congresso Brasileiro de Informática na Educação (CBIE 2016)

curriculares, programas e metodologias de ensino e de aprendizagem) ou em nível micro, no âmbito das unidades escolares e nas salas de aula de graduação.

A literatura científica possui diversos trabalhos que apresentam um conjunto de abordagens dialéticas que possuem significados e contextos distintos. Todavia, na Ciência

da Computação, os estudos explicitamente teórico-dialéticos são pouco difundidos. É necessário um trabalho muito cuidadoso para encontrar referências, ainda que sejam nas entrelinhas de artigos e teses, que apresentem elementos dialéticos na proposição de abordagens teóricas e/ou metodológicas da Ciência da Computação, mesmo em época de preocupação com estudos interdisciplinares (Souza, 2016).

Apesar do amplo espectro de perspectivas dialéticas, há poucas informações na literatura clássica da Ciência da Computação que subsidiem estudos sobre a relação entre esta ciência outras áreas ou linguagens, como as linguagens das artes, das ciências humanas, das ciências sociais, entre outras (Souza, 2016).

Tal fato demonstra a necessidade de estudos que possam levantar e discutir aspectos dialéticos para construção de conhecimento no campo da Computação que prescindam do uso das máquinas, uma vez que a Computação, assim como a Matemática, é uma ciência abstrata e fundamentalmente semiótica, cuja umas das suas principais funções é processar signos.

A Computação é uma ciência com origens na Matemática, onde residem as teorias fundamentais para os estudos computacionais. A Música e suas abstrações, por sua vez, guardam conexões com a Matemática, ao fundamentar-se em elementos como tempos, compassos, frequências, timbres, melodias, figuras musicais, entre outros. Vários conhecimentos musicais necessitam de raciocínio matemático para sua compreensão, como por exemplo o conhecimento de divisão de compassos e leitura rítmica, cuja compreensão passa pelo domínio das quatro operações matemáticas (Souza, 2016).

Se for possível relacionar elementos de raciocínios matemáticos e musicais, talvez seja igualmente possível conceber novos modos de compreensão da Computação a partir da Música e vice-versa. E por consequência, conceber novos modos de desenvolvimento do raciocínio computacional, em uma concepção mais compreensível para os estudantes.

\section{Metodologia de desenvolvimento da intervenção}

A Ação Curricular em Comunidade e em Sociedade (ACCS) é uma natureza especial de componente curricular optativo ofertado aos alunos de graduação e pós graduação da Universidade Federal da Bahia (UFBA), no qual são desenvolvidas ações de extensão no âmbito da tecnologia e inovação, favorecendo o compartilhamento e a produção de conhecimentos para e com a sociedade, numa perspectiva de transformação.

Dentre as possíveis ações desenvolvidas pela ACCS, está o Curso de Iniciação à Programação de Computadores (CIPC), cujo objetivo é apresentar noções iniciais de programação e desenvolver habilidades de programação para resolução de problemas por meio de algoritmos e do raciocínio computacional. 
V Congresso Brasileiro de Informática na Educação (CBIE 2016)

Anais dos Workshops do V Congresso Brasileiro de Informática na Educação (CBIE 2016)

No primeiro semestre de 2016 foram oferecidas três turmas do CIPC, todas voltadas a estudantes regularmente matriculados no ensino médio, ou recém-egressos, com conhecimentos em informática básica, mas sem conhecimentos de programação.

Os instrutores do CIPC foram estudantes de graduação voluntários (Bacharelado em Sistemas de Informação, Bacharelado Interdisciplinar em Ciência e Tecnologia e Bacharelado em Ciência da Computação).

Os cursos tiveram carga horária de 24 horas, distribuídas em 12 encontros semanais aos sábados. A intervenção a ser relatada ocorreu em apenas uma das turmas, cujas aulas ocorreram em um dos laboratórios de informática do Instituto de Matemática e Estatística da UFBA.

O planejamento do curso foi concebido colaborativamente com o envolvimento de instrutores e coordenadores, na expectativa de promover, além do aprendizado dos conteúdos de programação, condições favoráveis à aplicação e integração desses conhecimentos no dia a dia dos estudantes.

Por se tratar de um curso introdutório, a ementa foi composta pelos conteúdos de raciocínio lógico; algoritmos; declaração de variáveis e constantes; tipos de variáveis; instruções de entrada e saída; operadores aritméticos e lógicos; estruturas de controle; estruturas de repetição e vetores e algoritmos de ordenação. Utilizou-se ainda os ambientes Scratch $^{1}$ e VisuAlg ${ }^{2}$ (Souza, 2009).

Algoritmos de ordenação foi o último tema estudado pelas turmas. Portanto, todos os demais temas planejados já havia sido trabalhados em sala de aula. Dentre os algoritmos de ordenação, focalizou-se em apenas dois: Bubblesort e Mergesort. Todavia, a intervenção relatada neste artigo considerou somente o ensino do Mergesort.

Para realização da intervenção, a turma foi dividida aleatoriamente em dois grupos de sete estudantes, em salas distintas. Para o grupo A ministrou-se aula com uso de slides e elementos do raciocínio musical. As aulas para o grupo A foram ministradas por um estudante do Mestrado em Ciência da Computação, com formação em Licenciatura em Matemática e Licenciatura em Computação (em andamento). Para o grupo B, ministrou-se aula em formato tradicional, com uso de lousa branca, por um estudante concluinte do curso de Bacharelado em Ciência da Computação.

O grupo A utilizou um plano de aula com uma abordagem interdisciplinar, de título: Relação de Fração, Notas Musicais e Mergesort. Nesse plano, o objetivo foi fazer com que os estudantes a partir da ideia de fração pudessem utilizar elementos musicais (vide Figura 1) para a formação/construção da Escala Diatônica de Dó (C $^{2}$ - D - E - F - G A - B - C) e evidenciar a ideia de ordenação com base nas notas ordenadas da escala e que, intuitivamente, correlacionamos com raciocínio computacional, utilizando a concepção algorítmica do Mergesort para arrumar a escala quando desordenada (Algoritmo de Ordenação).

\footnotetext{
${ }^{1}$ https://scratch.mit.edu/

2 https://sourceforge.net/projects/visualg30/

${ }^{3}$ https://pt.wikipedia.org/wiki/Escala_diat\%C3\%B4nica
} 
V Congresso Brasileiro de Informática na Educação (CBIE 2016)

Anais dos Workshops do V Congresso Brasileiro de Informática na Educação (CBIE 2016)

Ao final das aulas, foi aplicada uma ficha de avaliação da aprendizagem com três questões sobre aplicação/simulação do algoritmo Mergesort aos dois grupos. Além disso, na aula seguinte, foi realizado um grupo focal com o objetivo de obter dos alunos as opiniões e impressões sobre o curso e a intervenção, com duração de aproximadamente 50 minutos.
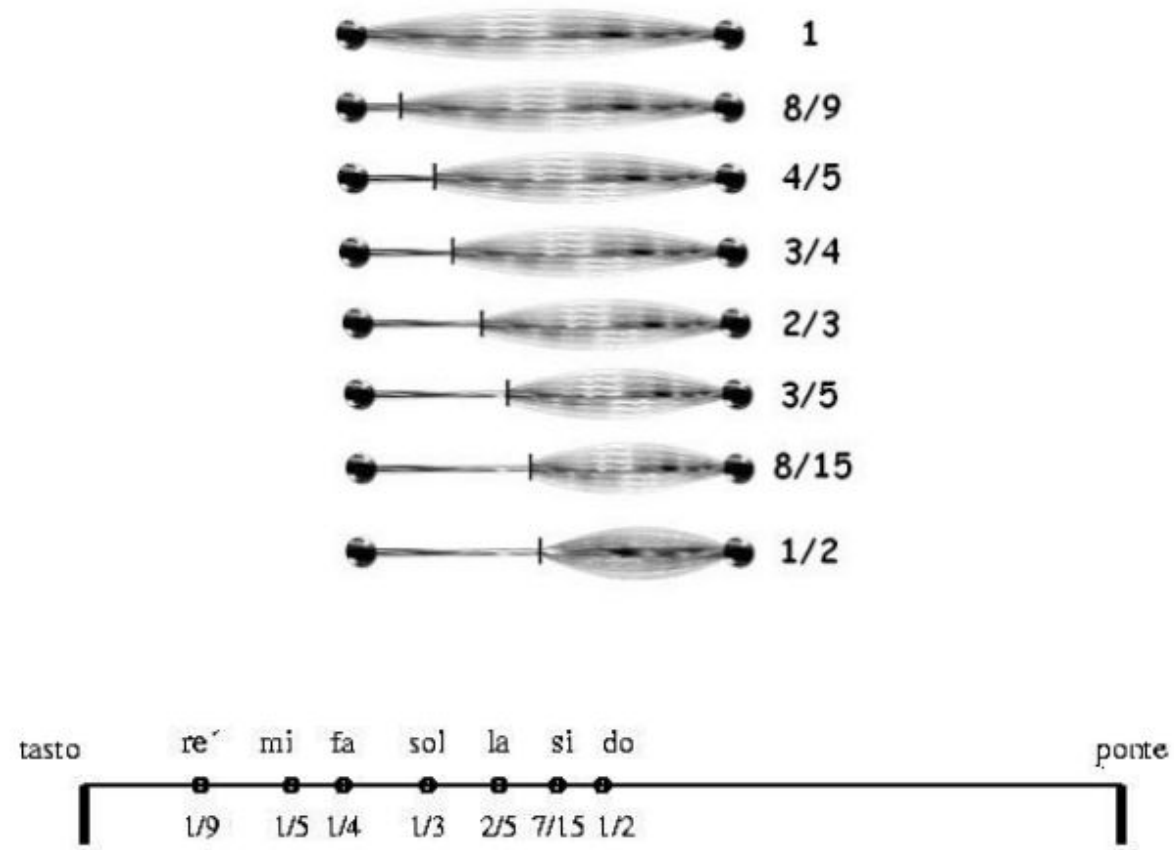

Figura 1. Notas da escala ordenada e sua relação com as frações e comprimentos de onda

Fonte: https://www.youtube.com/watch?v=6mHjdQpzxyI

\section{Análise dos resultados}

Com o objetivo de analisar as respostas obtidas nas avaliações, foi calculada a média aritmética dos resultados das notas obtidas pelos estudantes nas atividades. Observou-se diferença considerável entre os resultados das duas turmas. Em percentagem, o grupo A obteve um aproveitamento de 42,85\%, enquanto o grupo B alcançou aproximadamente o dobro, 85,71\%. Foi considerada, além da média geral das duas turmas, o resultado obtido por cada estudante, com o objetivo de entender o motivo dos erros que possa ter levado alguns deles a não pontuar na avaliação ou obter aproveitamento igual ou inferior a 50\%. Esses dados referentes à pontuação individual e geral da turma são devidamente expressos na Tabela 1.

Também foram identificadas as questões que obtiveram maior índice de erro ou de acerto entre os dois grupos. No grupo A, o maior índice de acerto foi na questão 01. Dos sete estudantes, quatro acertaram a questão. Quanto aos erros, a questão 02 obteve o maior índice, onde apenas dois estudantes responderam à questão corretamente. 
V Congresso Brasileiro de Informática na Educação (CBIE 2016)

Anais dos Workshops do V Congresso Brasileiro de Informática na Educação (CBIE 2016)

Tabela 1. Resultado individual e média por turma obtidos nas avaliações dado em percentuais.

\begin{tabular}{|c|r|c|r|}
\hline \multicolumn{2}{|c|}{ Grupo A } & \multicolumn{2}{c|}{ Grupo B } \\
\hline Estudante & Nota (\%) & Estudante & Nota (\%) \\
\hline A1 & 0,0 & B1 & 100,0 \\
\hline A2 & 50,0 & B2 & 100,0 \\
\hline A3 & 50,0 & B3 & 100,0 \\
\hline A4 & 0,0 & B4 & 100,0 \\
\hline A5 & 100,0 & B5 & 100,0 \\
\hline A6 & 0,0 & B6 & 50,0 \\
\hline A7 & 100,0 & B7 & 50,0 \\
\hline Aproveitamento (\%) & 42,86 & Aproveitamento (\%) & 85,71 \\
\hline
\end{tabular}

Por sua vez, dentre os resultados do grupo B, o maior índice de acerto foi na questão 02. Todos estudantes acertaram essa questão. A questão 01, apenas dois estudantes deste grupo não responderam corretamente, esse foi o maior índice de erro. No grupo A, apenas dois estudantes acertaram todas as questões, enquanto no grupo B, cinco estudantes alcançaram o mesmo resultado, conforme apresentado na Tabela 2.

Tabela 2. Tabela com o índice de erros e acertos por questão de cada turma.

\begin{tabular}{|r|r|r|}
\hline \multicolumn{3}{|c|}{ Grupo A } \\
\hline Questão & Acertos & Erros \\
\hline I & 4 & 3 \\
\hline II & 2 & 5 \\
\hline \multicolumn{3}{|c|}{ Grupo B } \\
\hline Questão & Acertos & Erros \\
\hline I & 5 & 2 \\
\hline II & 7 & 0 \\
\hline
\end{tabular}

De acordo com esses dados, podemos perceber que na primeira questão, o resultado não foi muito diferente entre as duas turmas. Contudo, na segunda questão, há nítida disparidade. Enquanto todos os estudante acertaram a questão 02 no grupo B, essa mesma questão obteve o maior índice de erro no grupo A. Podemos utilizar algumas hipóteses a 
V Congresso Brasileiro de Informática na Educação (CBIE 2016)

Anais dos Workshops do V Congresso Brasileiro de Informática na Educação (CBIE 2016)

fim de compreender quais fatores, combinados ou não, podem ter favorecido essas diferenças de resultado entre as turmas:

Critério I. Falta de clareza do enunciado: é possível que alguns estudantes não tenham entendido como responder à questão 02 . Este critério foi considerado devido a três respostas não condizerem com o esperado na questão. A priori, essas três respostas erradas ocorreram por conta da falta de entendimento por parte dos estudantes. A questão 02 requeria uma resposta combinada. Foram dadas quatro alternativas que deveriam completar as lacunas (passos do algoritmo Mergesort - representado por trechos de código), de acordo com a ordem escolhida pelo estudante. $\mathrm{Na}$ turma $\mathrm{A}$, dois estudantes escolheram apenas umas das alternativas e outro estudante escolheu duas alternativas repetindo-as de modo a preencher as lacunas. Logo, esses estudantes não identificaram que a questão solicitava o preenchimento das lacunas colocando os trechos de código na ordem correta.

Caso o enunciado da questão 02 não tenha sido elucidativa o suficiente, será preciso rever com mais cuidado os estilos de questões propostos nas atividades. Caberia, possivelmente, uma questão adicional em formulários de perfis de estudantes, com o intuito de saber antecipadamente o que eles entendem por questões objetivas, subjetivas, de múltipla escolha, dentre outras.

Critério II. Falta de entendimento do assunto: ainda é possível que os estudantes tenham entendido perfeitamente o que a questão 02 requeria; contudo, não conseguiram compreender o funcionamento do algoritmo. Essa possibilidade também não afetou o grupo $\mathrm{B}$, tendo em vista que todos responderam corretamente. Por sua vez, no grupo A os demais erros na referida questão podem ter sido por causa deste critério. A questão 03 pode indicar possível falta de entendimento acerca do algoritmo, tendo em vista que a mesma requeria uma explicação, sob o ponto de vista do estudante, sobre como eles chegaram à conclusão quanto à resposta da questão 02 .

Critério III: Interdisciplinaridade/Abordagem do professor: um dos objetivos da intervenção artigo foi avaliar se a interdisciplinaridade pode ser fator estimulador no processo de aprendizagem de conceitos computacionais; entretanto, há a possibilidade de que a combinação de conceitos de matemática, música e computação, nesta intervenção, não tenham surtido o efeito esperado. Não necessariamente pela interdisciplinaridade em si, mas pelo conjunto de elementos didáticos, inclusive pela abordagem utilizada pelos professores/instrutores.

Com base nas respostas livres da questão 03, foi possível observar se houve entendimento do assunto em algum grau. Embora a questão 03 estivesse diretamente relacionada à questão 02 , ela refletia também um possível entendimento acerca da primeira questão.

Questão 01: No grupo A, quatro estudantes acertaram e três erraram; enquanto no grupo B, cinco acertaram e dois erraram. Essa diferença entre as turmas é mínima, impossibilitando inferências precisas.

Questão 02: No grupo A, dois alunos acertaram a questão e cinco erraram. Aqueles que acertaram, também estão entre os que acertaram a questão 01. Além disso, suas respostas referentes à terceira questão condizem com o conteúdo. Com base nesses dados, concluiu-se que eles conseguiram entender o conteúdo aplicado. Entretanto, no grupo A, 
V Congresso Brasileiro de Informática na Educação (CBIE 2016)

Anais dos Workshops do V Congresso Brasileiro de Informática na Educação (CBIE 2016)

dos cinco estudantes que erraram, dois não responderam de forma coerente ao enunciado da questão. Das respostas à questão 03, por exemplo, esses cinco estudantes não demonstraram entendimento do conteúdo, onde dois deles responderam que escolheram determinadas opções simplesmente porque elas "se encaixavam".

Com base nesses dados, foi possível inferir que não houve um entendimento claro acerca do enunciado da questão para alguns, e para outros as respostas à questão 03 não refletiu compreensão do algoritmo ensinado em sala de aula.

No grupo B, todos os sete estudantes acertaram a questão. Além disso, na resposta à terceira questão, a maioria informou que seguiu a "lógica". Com base nesses dados, concluiu-se que não só o entendimento da questão correspondeu ao enunciado, mas também o entendimento do assunto foi alcançado.

Além da análise das atividades avaliativas, foi realizada uma análise a partir dos perfis registrados em dois questionários anteriormente aplicados, no momento de matrícula dos estudantes. Suas perguntas previam obter informações acerca do conhecimento prévio na área de computação, além do interesse em programação de computador e o de prosseguir na área.

No primeiro questionário haviam 12 questões de múltipla escolha. Cada acerto correspondeu a um ponto. A maior nota foi 10 (dez) (entre zero e 10). Enquanto a menor nota foi cinco. A média aritmética geral da turma foi de aproximadamente de 8,4 pontos. Com isso, foi possível concluir que a turma, no geral, ingressou no curso com conhecimentos básicos sobre computador e informática.

No segundo questionário, foram realizadas oito questões de múltipla escolha, contendo um campo para anotações de algum tipo de observação do entrevistador. Se a resposta de uma das perguntas fosse a esperada de um aluno com o perfil mais adequado para o curso de programação, além de suas experiências com programação e software livre, então o aluno recebia dois pontos pela questão. Se a resposta não fosse considerada adequada, o então candidato receberia apenas 01 ponto. Logo, 10 pontos seria uma pontuação razoável.

Nessa análise, com base nas notas do segundo questionário, apenas dois estudantes fizeram pontuação abaixo de 10 . Isto significa que a maioria dos estudantes estava familiarizada com os requerimentos para o curso, ainda que não conhecessem sobre programação.

Ao observar os resultados destes mesmos estudantes nas atividades avaliativas, nota-se que no grupo $\mathrm{A}$, o resultado não correspondeu ao esperado em concordância com os perfis. Porém no grupo B, os perfis refletem o resultado das provas. Este fato é mais um forte indício de que algum fator relacionado a abordagem utilizada pelo professor ao tratar com a interdisciplinaridade, pode ter comprometido o resultado de alguns estudantes.

O grupo focal, realizado na aula seguinte à aplicação da intervenção, ocorreu da seguinte forma: (i) identificou-se os alunos presentes e os pesquisadores; (ii) houve apresentação dos pesquisadores, da proposta e operacionalização do grupo focal; (iii) os pesquisadores explicaram a importância do Termo de Consentimento Livre e Esclarecido (TCLE), enfatizando o anonimato dos sujeitos e a importância da criticidade e participação deles nesta etapa. Nesse momento, foi acordado que cada participante ao iniciar sua fala deveria informar seu nome, para fins de transcrição do áudio. Uma das primeira perguntas 
V Congresso Brasileiro de Informática na Educação (CBIE 2016)

Anais dos Workshops do V Congresso Brasileiro de Informática na Educação (CBIE 2016)

foi sobre o motivo que os levaram a se candidatar em um curso de iniciação à programação. Entre respostas variadas, a maioria do grupo já tinha tido um contato superficial com a área ou uma curiosidade com o tema e por isso resolveram se inscrever. Apenas alguns entraram no curso sem interesse ou motivação inicial.

Os alunos demonstraram satisfação com as aulas ministradas, pois segundo eles: "não foi aquela coisa monótona". Assim, as aulas ministradas pelo Scratch e de forma mais dinâmica e informal foi bem recebida pelos alunos.

A maioria da turma informou que não conhecia algoritmos de ordenação (classificação), com exceção de um estudante, que afirmara reconhecer o nome "Mergesort", ainda que não saiba como ele funciona. Quando questionados sobre alguma possibilidade de aplicação do Mergesort, poucos conseguiram visualizar o Mergesort em uma situação prática do dia a dia.

Os estudantes do grupo A demonstraram-se satisfeitos com a aplicação, apresentando interesse na relação da música com a computação. Porém, eles informaram que o conteúdo principal - a "base" da ordenação - não foi focalizado. O grupo B mostrou-se satisfeito com a aula e um de seus estudantes comentou que conseguiu entender o conteúdo mesmo chegando atrasado no dia da aula.

$\mathrm{Na}$ resposta sobre como o Mergesort funciona, utilizando o exemplo da questão 01 da avaliação, os estudantes sublinharam a dificuldade de entendimento do enunciado da questão.

\section{Considerações finais e desafios futuros}

Esta experiência, ainda que piloto, trouxe à tona critérios e parâmetros importantes a serem considerados em uma próxima ação semelhante. Especialmente pela verificação de que a postura interdisciplinar per si não é suficiente para alcançar bons resultados educacionais.

Isto porque o resultado alcançado pelos estudantes participantes do grupo A (com articulação com raciocínio musical) não foi superior ao resultado do grupo B (sem articulação com raciocínio musical).

Todavia, durante o grupo focal, os estudantes participantes do grupo A foram categóricos em falar como a aula com elementos de raciocínio musical foi interessante e prendeu a atenção dos estudantes.

Sem o objetivo de esgotar o tema, nem de identificar resultados generalizáveis, acredita-se que possivelmente a abordagem didática do professor e sua experiência técnica e docente, para além do planejamento didático, seja elemento coadjuvante para o sucesso de uma iniciativa interdisciplinar.

Eis um dos desafios que surge neste ínterim a partir desta experiência: promover melhor preparação dos professores, aliada a uma abordagem interdisciplinar e dialética mais consistente.

\section{Agradecimentos}

Os autores agradecem à Fundação de Amparo à Pesquisa do Estado da Bahia (FAPESB) e à Pró-Reitoria de Extensão Universitária da Universidade Federal da Bahia (UFBA) pelo apoio financeiro a este estudo. 
V Congresso Brasileiro de Informática na Educação (CBIE 2016)

Anais dos Workshops do V Congresso Brasileiro de Informática na Educação (CBIE 2016)

\section{Referências}

Cassel, L.N. (2011) Interdisciplinary computing is the answer: now, what was the question?. Inroads, vol. 2, issue 1. p. 4-6.

Fazenda, I.C.A. (2010) Interdisciplinaridade e Transdisciplinaridade na formação de Professores. Ideação (Unioeste), v. 10, n. 1, p. 93-103.

França, R. S.; Amaral, H. J. C. (2013) Ensino de Computação na Educação Básica no Brasil: Um Mapeamento Sistemático. XXI Workshop sobre Educação em Computação (WEI 2013). Anais do XXXIII Congresso da Sociedade Brasileira de Computação. p. 426-431.

Freire, P. (1996) Pedagogia da Autonomia: saberes necessários à prática educativa. São Paulo: Paz e Terra.

Hirotaka, A. (2014) Anybody can learn: computer science is not a foreign language. Code.org. http://blog.code.org/post/75129943201/language.. Acessado em 25 de fevereiro de 2015.

Matos, E.; Paiva, F; Corlett, E. (2016) "Novas atividades de computação desplugada para promoção de integração curricular na escola.”. In: Raabe, A.L.A; Gomes, A.S.; Bittencourt, I.I.; Pontual,T. (org.). Educação Criativa: multiplicando experiências para a aprendizagem, Recife: Pipa Comunicação, 2016. p. 205-251.

Paiva, L.F, Ferreira, A.C., Rocha, C., Barreto, J.S., Lopes, R. H., Melhor, A., Matos, E. (2015). Uma Experiência Piloto de Integração Curricular do Raciocínio Computacional na Educação Básica. I Workshop de Ensino em Pensamento Computacional, Algoritmos e Programação (WAlgProg 2015). Maceió-AL. Anais dos Workshops do CBIE 2015. Maceió - AL. Porto Alegre: SBC. p. 1300-1309.

Plaza, J. (1987) Tradução intersemiótica. São Paulo: Perspectiva.

Ribeiro, L.; Nunes, D.; Cruz, M.; Matos, E. (2014) Computational Thinking: possibilities and challenges. In: Post-Proceedings of 2nd Workshop-School on Theoretical Computer Science. 1. ed. Los Alamitos: IEEE. p. 22-25.

Sancho, J.M. (2006) Tecnologias da informação e comunicação a recursos educativos.In: SANCHO, Juana Maria \& HERNÁNDEZ, Fernando (Org.). Tecnologias para transformar a educação. Porto alegre: Artmed.

Souza, C.M. (2009) "VisuAlg - Ferramenta de Apoio ao Ensino de Programação". In: Revista TECCEN, v. 2, n. 2, setembro. p. 10.

Souza, N.S. (2016) Uma Abordagem Dialética para a Pesquisa Interdisciplinar em Interação Humano-Computador. Dissertação (Mestrado em Ciência da Computação). Universidade Federal da Bahia, Salvador.

Warschauer, C.A (2000) Roda e o Resgate da Fala Adormecida: buscando caminhos para a interdisciplinaridade nas séries iniciais. In: Ivani Fazenda. (Org.). A academia vai à escola. Campinas -SP: Papirus, 1995, v.1. p. 41-50. 